\title{
OTHER ARTICLES NOTED (25 Apr 2003 to 18 Jul 2003)
}

A list of the journals that are reviewed and the criteria for selecting articles from these journals for inclusion in Evidence-Based Nursing are included in each issue. The following articles passed all criteria but were not abstracted because, in the judgment of the editors, their findings were less widely applicable to nursing practice, the topic was of interest to only a select group of nurse specialists, or the topic was recently addressed in another abstract.

\section{Treatment}

Alhasso A, Glazener CMA, Pickard R, et al. Adrenergic drugs for urinary incontinence in adults. Cochrane Database Syst Rev 2003;(2):CD001842. http://www.ncbi.nlm.nih.gov/entrez/ query.fcgi? $\mathrm{cmd}=$ Retrieve $\& \mathrm{db}=$ PubMed\&list_uids $=12804414 \&$ $\mathrm{dopt}=\mathrm{Abstract}$

Allison JE, Hurley LB, Hiatt RA, et al. A randomized controlled trial of test-and-treat strategy for Helicobacter pylori: clinical outcomes and health care costs in a managed care population receiving long-term acid suppression therapy for physiciandiagnosed peptic ulcer disease. Arch Intern Med 2003;163: 1165-71. http://www.ncbi.nlm.nih.gov/entrez/query.fcgi?.cmd= Retrieve\&db=PubMed\&list_uids $=12767952 \& d o p t=A b s t r a c t$

Amato L, Davoli M, Ferri M, et al. Methadone at tapered doses for the management of opioid withdrawal. Cochrane Database Syst Rev 2003;(2):CD003409. http://www.ncbi.nlm.nih.gov/ entrez/query.fcgi? cmd=Retrieve\&db=PubMed\&list_uids= $12804464 \&$ dopt=Abstract

Andersen BR, Kallehave FL, Andersen HK. Antibiotics versus placebo for prevention of postoperative infection after appendicectomy. Cochrane Database Syst Rev 2003;(2):CD001439. http:// www.ncbi.nlm.nih.gov/entrez/query.fcgi?cmd=Retrieve\&db= PubMed\&list_uids=12804408\&dopt=Abstract

Anderson GC, Moore E, Hepworth J, et al. Early skin-to-skin contact for mothers and their healthy newborn infants. Cochrane Database Syst Rev 2003;(2):CD003519. http://www.ncbi.nlm.nih. gov/entrez/query.fcgi?cmd=Retrieve\& $\mathrm{db}=$ PubMed\&list_uids $=$ $12804473 \&$ dopt $=$ Abstract

Ascott-Evans BH, Guanabens N, Kivinen S, et al. Alendronate prevents loss of bone density associated with discontinuation of hormone replacement therapy: a randomized controlled trial. Arch Intern Med 2003;163:789-94. http://www.ncbi.nlm.nih. gov/entrez/query.fcgi? $\mathrm{cmd}=$ Retrieve $\& \mathrm{db}=$ PubMed\&list_uids $=$ 12695269\&dopt=Abstract

Assendelft WJ, Morton SC, Yu EI, et al. Spinal manipulative therapy for low back pain. A meta-analysis of effectiveness relative to other therapies. Ann Intern Med 2003;138:871-81. http://www.ncbi.nlm.nih.gov/entrez/query.fcgi?cmd=Retrieve $\& \mathrm{db}=$ PubMed\&list_uids $=12779297 \& \mathrm{dopt}=$ Abstract

Balaban DH, Leavell BS, Oblinger MJ, et al. Low volume bowel preparation for colonoscopy: randomized, endoscopist-blinded trial of liquid sodium phosphate versus tablet sodium phosphate. Am J Gastroenterol 2003;98:827-32. http://www.ncbi.nlm. nih.gov/entrez/query.fcgi? $\mathrm{cmd}=$ Retrieve $\& \mathrm{db}=$ PubMed\& list_uids=12738463\&dopt=Abstract

Balaguer A, Escribano J, Roque M. Infant position in neonates receiving mechanical ventilation. Cochrane Database Syst Rev 2003;(2):CD003668. http://www.ncbi.nlm.nih.gov/entrez/query. fcgi?cmd=Retrieve $\& d b=$ PubMed\&list_uids $=12804483 \& d o p t=$ Abstract
Barnett AH, Grant PJ, Hitman GA, et al. Rosiglitazone in type 2 diabetes mellitus: an evaluation in British Indo-Asian patients. Diabet Med 2003;20:387-93. http://www.ncbi.nlm.nih.gov/entrez/ query.fcgi? $\mathrm{cmd}=$ Retrieve $\& \mathrm{db}=\mathrm{PubMed} \&$ clist_uids $=12752488 \&$ dopt=Abstract

Becker C, Kron M, Lindemann U, et al. Effectiveness of a multifaceted intervention on falls in nursing home residents. $J \mathrm{Am}$ Geriatr Soc 2003;51:306-13. http://www.ncbi.nlm.nih.gov/entrez/ query.fcgi? $\mathrm{cmd}=$ Retrieve\&db=PubMed\&list_uids=12588573\&dopt $=$ Abstract

Berliner E, Ozbilgin B, Zarin DA. A systematic review of pneumatic compression for treatment of chronic venous insufficiency and venous ulcers. J Vasc Surg 2003;37:539-44. http:// www.ncbi.nlm.nih.gov/entrez/query.fcgi? $\mathrm{cmd}=$ Retrieve $\& \mathrm{db}=$ PubMed\&list_uids=12618689\&dopt=Abstract

Berseth CL, Bisquera JA, Paje VU. Prolonging small feeding volumes early in life decreases the incidence of necrotizing enterocolitis in very low birth weight infants. Pediatrics 2003; 111:529-34. http://www.ncbi.nlm.nih.gov/entrez/query.fcgi?cmd $=$ Retrieve $\& d b=$ PubMed\&list_uids $=12612232 \& d o p t=$ Abstract

Bhandari N, Bahl R, Mazumdar S, et al. Effect of communitybased promotion of exclusive breastfeeding on diarrhoeal illness and growth: a cluster randomised controlled trial. Lancet 2003;361:1418-23. http://www.ncbi.nlm.nih.gov/entrez/query .fcgi?cmd=Retrieve\&db=PubMed\&list_uids=12727395\&dopt= Abstract

Binder EF, Schechtman KB, Ehsani AA, et al. Effects of exercise training on frailty in community-dwelling older adults: results of a randomized, controlled trial.J Am Geriatr Soc 2002;50:1921-8. http://www.ncbi.nlm.nih.gov/entrez/query.fcgi?cmd=Retrieve $\& d b=$ PubMed\&list_uids=12473001\&dopt=Abstract

Black HR, Elliott WJ, Grandits G, et al. Principal results of the Controlled Onset Verapamil Investigation of Cardiovascular End Points (CONVINCE) trial. JAMA 2003;289:2073-82. http://www.ncbi.nlm.nih.gov/entrez/query.fcgi?cmd=Retrieve $\& \mathrm{db}=$ PubMed\&list_uids=12709465\&dopt=Abstract

Boustani M, Peterson B, Hanson L, et al. Screening for dementia in primary care: a summary of the evidence for the U.S. Preventive Services Task Force. Ann Intern Med 2003;138:927-37. http://www.ncbi.nlm.nih.gov/entrez/query.fcgi?cmd=Retrieve $\& \mathrm{db}=$ PubMed\&list_uids=12779304\&dopt=Abstract

Bravata DM, Sanders L, Huang J, et al. Efficacy and safety of low-carbohydrate diets: a systematic review. JAMA 2003;289: 1837-50. http://www.ncbi.nlm.nih.gov/entrez/query.fcgi?cmd $=$ Retrieve $\& d b=$ PubMed\&list_uids=12684364\&dopt=Abstract 
Brodaty H, Green A, Koschera A. Meta-analysis of psychosocial interventions for caregivers of people with dementia. J Am Geriatr Soc 2003;51:657-64. http://www.ncbi.nlm.nih.gov/entrez/ query.fcgi? $\mathrm{cmd}=$ Retrieve $\& \mathrm{db}=$ PubMed\&list_uids $=12752841 \&$ $\mathrm{dopt}=\mathrm{Abstract}$

Brown A, Taylor R, Noorani H, et al. Exercise-based cardiac rehabilitation programs for coronary artery disease: a systematic clinical and economic review. Ottawa: Canadian Coordinating Office for Health Technology Assessment (CCOHTA), Mar 2003. www.ccohta.ca

Cherkin DC, Sherman KJ, Deyo RA, et al. A review of the evidence for the effectiveness, safety, and cost of acupuncture, massage therapy, and spinal manipulation for back pain. Ann Intern Med 2003;138:898-906. http://www.ncbi.nlm.nih.gov/ entrez/query.fcgi? $c$ cmd=Retrieve\&db=PubMed\&list_uids= 12779300\&dopt=Abstract

Chobanian AV, Bakris GL, Black HR, et al. The Seventh Report of the Joint National Committee on Prevention, Detection, Evaluation, and Treatment of High Blood Pressure: the JNC 7 report.JAMA 2003;289:2560-72. http://www.ncbi.nlm.nih.gov/ entrez/query.fcgi? $\mathrm{cmd}=$ Retrieve\&db=PubMed\&list_uids= $127481991 \&$ dopt=Abstract

Colak T, Ipek T, Kanik A, et al. Efficacy of topical nonsteroidal antiinflammatory drugs in mastalgia treatment. J Am Coll Surg 2003;196:525-30. http://www.ncbi.nlm.nih.gov/entrez/query. fcgi? $c$ dend=Retrieve\&db=PubMed\&list_uids=12691925\&dopt= Abstract

Collins R, Armitage J, Parish S, et al. MRC/BHF Heart Protection Study of cholesterol-lowering with simvastatin in 5963 people with diabetes: a randomised placebo-controlled trial. Lancet 2003;361:2005-16. http://www.ncbi.nlm.nih.gov/ entrez/query.fcgi? $\mathrm{cmd}=$ Retrieve\&db=PubMed\&list_uids= 12814710\&dopt=Abstract

Colom F, Vieta E, Martinez-Aran A, et al. A randomized trial on the efficacy of group psychoeducation in the prophylaxis of recurrences in bipolar patients whose disease is in remission. Arch Gen Psychiatry 2003;60:402-7. http://www.ncbi.nlm.nih. gov/entrez/query.fcgi?cmd=Retrieve $\& \mathrm{db}=$ PubMed\&list_uids $=$ 12695318\&dopt=Abstract

Conde-Agudelo A, Diaz-Rossello JL, Belizan JM. Kangaroo mother care to reduce morbidity in low birthweight infants. Cochrane Database Syst Rev 2003;(2):CD002771. http:// www.ncbi.nlm.nih.gov/entrez/query.fcgi?cmd=Retrieve $\& \mathrm{db}=$ PubMed\&list_uids=12804436\&dopt=Abstract

Cooper PJ, Murray L, Wilson A, et al. Controlled trial of the short- and long-term effect of psychological treatment of postpartum depression. I. Impact on maternal mood. Br J Psychiatry 2003;182:412-9. http://www.ncbi.nlm.nih.gov/entrez/query. fcgi? $c$ d =Retrieve\&db=PubMed\&list_uids=12724244\&dopt= Abstract

Copeland LA, Blow FC, Barry KL. Health care utilization by older alcohol-using veterans: effects of a brief intervention to reduce at-risk drinking. Health Educ Behav 2003;30:305-21.

Criscuoli G, D'Amora S, Ripa G, et al. Frequency of surgery among children who have adenotonsillar hypertrophy and improve after treatment with nasal beclomethasone. Pediatrics 2003;111:e236-8. http://www.ncbi.nlm.nih.gov/entrez/query. fcgi? $c m d=$ Retrieve\&db=PubMed\&list_uids $=12612277 \& d o p t=$ Abstract

Curry SJ, Ludman EJ, Graham E, et al. Pediatric-based smoking cessation intervention for low-income women: a randomized trial. Arch Pediatr Adolesc Med 2003;157:295-302. http:// www.ncbi.nlm.nih.gov/entrez/query.fcgi? $\mathrm{cmd}=$ Retrieve\&db= PubMed\&list_uids=12622686\&dopt=Abstract

Davis PG, Henderson-Smart DJ. Nasal continuous positive airways pressure immediately after extubation for preventing morbidity in preterm infants. Cochrane Database Syst Rev 2003; (2):CD000143. http://www.ncbi.nlm.nih.gov/entrez/query.fcgi? cmd=Retrieve\&db=PubMed\&list_uids=12804388\&dopt= Abstract

Delaney BC, Moayyedi P, Forman D. Initial management strategies for dyspepsia. Cochrane Database Syst Rev 2001;(3):CD001961. http://www.ncbi.nlm.nih.gov/entrez/ query.fcgi? $\mathrm{cmd}=$ Retrieve $\& \mathrm{db}=$ PubMed\&list_uids $=12804417 \&$ dopt $=$ Abstract

DeWitt DE, Hirsch IB. Outpatient insulin therapy in type 1 and type 2 diabetes mellitus: scientific review. JAMA 2003;289: 2254-64. http://www.ncbi.nlm.nih.gov/entrez/query.fcgi?.cmd= Retrieve\&db=PubMed\&list_uids=12734137\&dopt=Abstract

Díaz-Gómez NM, Doménech E, Barroso F, et al. The effect of zinc supplementation on linear growth, body composition, and growth factors in preterm infants. Pediatrics 2003;111:1002-9. http://www.ncbi.nlm.nih.gov/entrez/query.fcgi?cmd=Retrieve $\& \mathrm{db}=$ PubMed\&list_uids $=12728080 \&$ dopt $=$ Abstract

Di Loreto C, Fanelli C, Lucidi P, et al. Validation of a counseling strategy to promote the adoption and the maintenance of physical activity by type 2 diabetic subjects. Diabetes Care 2003; 26:404-8. http://www.ncbi.nlm.nih.gov/entrez/query.fcgi?cmd= Retrieve\&db=PubMed\&list_uids $=12547870 \&$ dopt $=$ Abstract

Donta ST, Clauw DJ, Engel CC Jr, et al. Cognitive behavioral therapy and aerobic exercise for Gulf War veterans' illnesses: a randomized controlled trial. JAMA 2003;289:1396-404. http:// www.ncbi.nlm.nih.gov/entrez/query.fcgi? cmd=Retrieve\&db= PubMed\&list_uids=12636462\&dopt=Abstract

Drblik S, Lapierre G, Thivierge R, et al. Comparative efficacy of terbutaline sulphate delivered by Turbuhaler dry powder inhaler or pressurised metered dose inhaler with Nebuhaler spacer in children during an acute asthmatic episode. Arch Dis Child 2003;88:319-23. http://www.ncbi.nlm.nih.gov/entrez/ query.fcgi? cmd=Retrieve\&db=PubMed\&list_uids $=12651757 \&$ dopt=Abstract

Ducharme FM. Inhaled glucocorticoids versus leukotriene receptor antagonists as single agent asthma treatment: systematic review of current evidence. BMJ 2003;326:621-3. http:// www.ncbi.nlm.nih.gov/entrez/query.fcgi? $\mathrm{cmd}=$ Retrieve $\& \mathrm{db}=$ PubMed\&list_uids=12649233\&dopt=Abstract

Durham RC, Guthrie M, Morton RV, et al. Tayside-Fife clinical trial of cognitive-behavioural therapy for medication-resistant psychotic symptoms. Results to 3-month follow-up. Br J Psychiatry 2003;182:303-11. http://www.ncbi.nlm.nih.gov/entrez/query. fcgi?cmd=Retrieve\&db=PubMed\&list_uids=12668405\&dopt= Abstract 
Edwards D, Burnard P. A systematic review of stress and stress management interventions for mental health nurses. J Adv Nurs 2003;42:169-200. http://www.ncbi.nlm.nih.gov/entrez/query. fcgi?cmd=Retrieve\&db=PubMed\&list_uids=12670386\&dopt= Abstract

El-Bassel N, Witte SS, Gilbert L, et al. The efficacy of a relationship-based HIV/STD prevention program for heterosexual couples. Am J Public Health 2003;93:963-9. http:// www.ncbi.nlm.nih.gov/entrez/query.fcgi?cmd=Retrieve\&db= PubMed\&list_uids=12773363\&dopt=Abstract

Ellis P, Robinson P, Ciliska D, et al. Diffusion and dissemination of evidence-based cancer control interventions. Evidence Report/ Technology Assessment Number 79. AHRQ Publication No. 03-E033. Rockville, MD: Agency for Healthcare Research and Quality, May 2003. http://www.ahrq.gov/clinic/epcsums/ canconsum.htm

Elley CR, Kerse N, Arroll B, et al. Effectiveness of counselling patients on physical activity in general practice: cluster randomised controlled trial. BMJ 2003;326:793-6. http:// www.ncbi.nlm.nih.gov/entrez/query.fcgi?cmd=Retrieve\&db= PubMed\&list_uids=12689976\&dopt=Abstract

Fals-Stewart W, O'Farrell TJ. Behavioral family counseling and naltrexone for male opioid-dependent patients. J Consult Clin Psychol 2003;71:432-42. http://www.ncbi.nlm.nih.gov/entrez/ query.fcgi?.cmd=Retrieve\&db=PubMed\&list_uids $=12795568 \&$ dopt=Abstract

Fritsche A, Schweitzer MA, Häring HU. Glimepiride combined with morning insulin glargine, bedtime neutral protamine hagedorn insulin, or bedtime insulin glargine in patients with type 2 diabetes. A randomized, controlled trial. Ann Intern Med 2003;138:952-9. http://www.ncbi.nlm.nih.gov/entrez/query. fcgi?cmd=Retrieve\&db=PubMed\&list_uids=12809451\&dopt= Abstract

Forna F, Gulmezoglu AM. Interventions for treating trichomoniasis in women. Cochrane Database Syst Rev 2003;(2):CD000218. http://www.ncbi.nlm.nih.gov/entrez/query.fcgi?cmd=Retrieve $\& d b=$ PubMed\&list_uids=12804391\&dopt=Abstract

Gallagher R, McKinley S, Dracup K. Effects of a telephone counseling intervention on psychosocial adjustment in women following a cardiac event. Heart Lung 2003;32:79-87. http:// www.ncbi.nlm.nih.gov/entrez/query.fcgi? $c \mathrm{dm}=$ Retrieve\&db= PubMed\&list_uids=12734530\&dopt=Abstract

Gené E, Calvet X, Azagra R, et al. Triple vs. quadruple therapy for treating Helicobacter pylori infection: a meta-analysis. Aliment Pharmacol Ther 2003;17:1137-43. http:// www.ncbi.nlm.nih.gov/entrez/query.fcgi?cmd=Retrieve\&db= PubMed\&list_uids=12752350\&dopt=Abstract

Goldhaber-Fiebert JD, Goldhaber-Fiebert SN, Tristán ML, et al. Randomized controlled community-based nutrition and exercise intervention improves glycemia and cardiovascular risk factors in type 2 diabetic patients in rural Costa Rica. Diabetes Care 2003;26:24-9. http://www.ncbi.nlm.nih.gov/entrez/query. fcgi? $\mathrm{cmd}=$ Retrieve $\& d b=$ PubMed\&list_uids $=12502654 \& d o p t=$ Abstract
Guenaga KF, Matos D, Castro AA, et al. Mechanical bowel preparation for elective colorectal surgery. Cochrane Database Syst Rev 2003;(2):CD001544. http://www.ncbi.nlm.nih.gov/entrez/ query.fcgi? $\mathrm{cmd}=$ Retrieve\&db=PubMed\&list_uids=12804412\&dopt $=$ Abstract

Halpern MT, Khan ZM, Stanford RH, et al. Asthma: resource use and costs for inhaled corticosteroid vs leukotriene modifier treatment-a meta-analysis. J Fam Pract 2003;52:382-9. http:// www.ncbi.nlm.nih.gov/entrez/query.fcgi?cmd=Retrieve\&db= PubMed\&list_uids=12737772\&dopt=Abstract

Hays J, Ockene JK, Brunner RL, et al. Effects of estrogen plus progestin on health-related quality of life. $N$ Engl J Med 2003;348:1839-54. http://www.ncbi.nlm.nih.gov/entrez/query. fcgi? $c m d=$ Retrieve\&db=PubMed\&list_uids $=12642637 \&$ dopt $=$ Abstract

Heh SS, Fu YY. Effectiveness of informational support in reducing the severity of postnatal depression in Taiwan. J Adv Nurs 2003;42:30-6. http://www.ncbi.nlm.nih.gov/entrez/query. fcgi? $\mathrm{cmd}=$ Retrieve\&db=PubMed\&list_uids $=12641809 \& \mathrm{cdopt}=$ Abstract

Henggeler SW, Rowland MD, Halliday-Boykins C, et al. One-year follow-up of multisystemic therapy as an alternative to the hospitalization of youths in psychiatric crisis. J Am Acad Child Adolesc Psychiatry 2003;42:543-51. http://www.ncbi.nlm.nih. gov/entrez/query.fcgi? $\mathrm{cmd}=$ Retrieve $\& \mathrm{db}=$ PubMed\&list_uids $=$ 12707558\&dopt=Abstract

Herbison P, Hay-Smith J, Ellis G, et al. Effectiveness of anticholinergic drugs compared with placebo in the treatment of overactive bladder: systematic review. BMJ 2003;326:841-4. http://www.ncbi.nlm.nih.gov/entrez/query.fcgi?cmd=Retrieve\&db $=$ PubMed\&list_uids=12702614\&dopt=Abstract

Highland KB, Strange C, Heffner JE. Long-term effects of inhaled corticosteroids on $\mathrm{FEV}_{1}$ in patients with chronic obstructive pulmonary disease. A meta-analysis. Ann Intern Med 2003;138:969-73. http://www.ncbi.nlm.nih.gov/entrez/query. fcgi? $c$ de $=$ Retrieve $\& d b=$ PubMed\&list_uids $=12809453 \&$ dopt $=$ Abstract

Hignett S. Systematic review of patient handling activities starting in lying, sitting and standing positions. $J$ Adv Nurs 2003;41:545-52. http://www.ncbi.nlm.nih.gov/entrez/query. fcgi? $\mathrm{cmd}=$ Retrieve\&db=PubMed\&list_uids $=12622863 \& d o p t=$ Abstract

Hooper L, Bartlett C, Davey Smith G, et al. Reduced dietary salt for prevention of cardiovascular disease. Cochrane Database Syst Rev 2003;(2):CD003656. http://www.ncbi.nlm.nih.gov/entrez/ query.fcgi? $\mathrm{cmd}=$ Retrieve $\& \mathrm{db}=$ PubMed\&list_uids $=12804482 \&$ $\mathrm{dopt}=$ Abstract

Howard CR, Howard FM, Lanphear B, et al. Randomized clinical trial of pacifier use and bottle-feeding or cupfeeding and their effect on breastfeeding. Pediatrics 2003;111:511-8. http:// www.ncbi.nlm.nih.gov/entrez/query.fcgi?cmd=Retrieve\&db= PubMed\&list_uids=12612229\&dopt=Abstract

Hughes JR, Stead LF, Lancaster T. Antidepressants for smoking cessation. Cochrane Database Syst Rev 2003;(2):CD000031. http://www.ncbi.nlm.nih.gov/entrez/query.fcgi?cmd=Retrieve $\& \mathrm{db}=$ PubMed\&list_uids=12804385\&dopt=Abstract 
Hughes R, Goldkorn A, Masoli M, et al. Use of isotonic nebulised magnesium sulphate as an adjuvant to salbutamol in treatment of severe asthma in adults: randomised placebo-controlled trial. Lancet 2003;361:2114-7.http://www.ncbi.nlm.nih.gov/entrez/ query.fcgi? $\mathrm{cmd}=$ Retrieve\&db=PubMed\&list_uids $=12826434 \&$ dopt=Abstract

Huibers MJH, Beurskens AJHM, Bleijenberg G, et al. The effectiveness of psychosocial interventions delivered by general practitioners. Cochrane Database Syst Rev 2003;(2):CD003494. http://www.ncbi.nlm.nih.gov/entrez/query.fcgi?cmd=Retrieve $\& \mathrm{db}=$ PubMed\&list_uids=12804471\&dopt=Abstract

Izquierdo RE, Knudson PE, Meyer S, et al. A comparison of diabetes education administered through telemedicine versus in person. Diabetes Care 2003;26:1002-7. http://www.ncbi.nlm. nih.gov/entrez/query.fcgi? $\mathrm{cmd}=$ Retrieve\&db=PubMed\&list_uids= $12663564 \&$ dopt=Abstract

Kaneko Y, Floras JS, Usui K, et al. Cardiovascular effects of continuous positive airway pressure in patients with heart failure and obstructive sleep apnea. N Engl J Med 2003;348:1233-41. http://www.ncbi.nlm.nih.gov/entrez/query.fcgi?cmd=Retrieve $\& \mathrm{db}=$ PubMed\&list_uids=12660387\&dopt=Abstract

Kapczinski F, Lima MS, Souza JS, et al. Antidepressants for generalized anxiety disorder. Cochrane Database Syst Rev 2003;(2):CD003592. http://www.ncbi.nlm.nih.gov/entrez/ query.fcgi? $\mathrm{cmd}=$ Retrieve\&db=PubMed\&list_uids $=12804478 \&$ dopt=Abstract

Karjalainen K, Malmivaara A, van Tulder M, et al. Multidisciplinary biopsychosocial rehabilitation for neck and shoulder pain among working age adults. Cochrane Database Syst Rev 2003;(2):CD002194. http://www.ncbi.nlm.nih.gov/entrez/ query.fcgi? $\mathrm{cmd}=$ Retrieve\&db=PubMed\&list_uids $=12804428 \&$ dopt=Abstract

Karjalainen K, Malmivaara A, van Tulder M, et al. Multidisciplinary biopsychosocial rehabilitation for subacute low back pain among working age adults. Cochrane Database Syst Rev 2003;(2):CD002193. http://www.ncbi.nlm.nih.gov/entrez/ query.fcgi?cmd=Retrieve\&db=PubMed\&list_uids=12804427\& dopt=Abstract

Keenan SP, Sinuff T, Cook DJ, et al. Which patients with acute exacerbation of chronic obstructive pulmonary disease benefit from noninvasive positive-pressure ventilation? A systematic review of the literature. Ann Intern Med 2003;138:861-70. http://www.ncbi.nlm.nih.gov/entrez/query.fcgi?cmd= Retrieve\&db=PubMed\&list_uids=12779296\&dopt=Abstract

Kirk A, Mutrie N, MacIntyre P, et al. Increasing physical activity in people with type 2 diabetes. Diabetes Care 2003;26:1186-92. http://www.ncbi.nlm.nih.gov/entrez/query.fcgi? $\mathrm{cmd}=$ Retrieve\&db=PubMed\&list_uids=12663595\&dopt=Abstract

Koo WW, Hammami M, Margeson DP, et al. Reduced bone mineralization in infants fed palm olein-containing formula: a randomized, double-blinded, prospective trial. Pediatrics 2003;111:1017-23. http://www.ncbi.nlm.nih.gov/entrez/query. fcgi? $c$ cmd=Retrieve\&db=PubMed\&list_uids=12728082\&dopt= Abstract

Kovacs MJ, Rodger M, Anderson DR, et al. Comparison of 10-mg and 5-mg warfarin initiation nomograms together with low-molecular-weight heparin for outpatient treatment of acute venous thromboembolism. A randomized, double-blind, controlled trial. Ann Intern Med 2003;138:714-9. http://www.ncbi. nlm.nih.gov/entrez/query.fcgi? $\mathrm{cmd}=$ Retrieve\&db=PubMed\& list_uids $=12729425 \&$ dopt $=$ Abstract

Krishna S, Francisco BD, Balas EA, et al. Internet-enabled interactive multimedia asthma education program: a randomized trial. Pediatrics 2003;111:503-10. http://www.ncbi.nlm.nih.gov/entrez/ query.fcgi?cmd=Retrieve\&db=PubMed\&list_uids=12612228\&dopt $=$ Abstract

Kwekkeboom KL. Music versus distraction for procedural pain and anxiety in patients with cancer. Oncol Nurs Forum 2003;30:433-40. http://www.ncbi.nlm.nih.gov/entrez/query. fcgi? $c m d=$ Retrieve\&db=PubMed\&list_uids=12719743\&dopt= Abstract

Lai KC, Lau CS, Ip WY, et al. Effect of treatment of Helicobacter pylori on the prevention of gastroduodenal ulcers in patients receiving long-term NSAIDs: a double-blind, placebocontrolled trial. Aliment Pharmacol Ther 2003;17:799-805. http://www.ncbi.nlm.nih.gov/entrez/query.fcgi?cmd=Retrieve $\& \mathrm{db}=$ PubMed\&list_uids $=12641502 \& d o p t=$ Abstract

Laramee AS, Levinsky SK, Sargent J, et al. Case management in a heterogeneous congestive heart failure population: a randomized controlled trial. Arch Intern Med 2003;163:809-17. http://www.ncbi.nlm.nih.gov/entrez/query.fcgi? cmd=Retrieve $\& d b=$ PubMed\&list_uids=12695272\&dopt=Abstract

Latham N, Anderson C, Bennett D, et al. Progressive resistance strength training for physical disability in older people. Cochrane Database Syst Rev 2003;(2):CD002759. http://www.ncbi.nlm. nih.gov/entrez/query.fcgi?.cmd=Retrieve\&db=PubMed\&list_uids $=12804434 \&$ dopt $=$ Abstract

Leucht S, Wahlbeck K, Hamann J, et al. New generation antipsychotics versus low-potency conventional antipsychotics: a systematic review and meta-analysis. Lancet 2003;361:1581-9. http://www.ncbi.nlm.nih.gov/entrez/query.fcgi?cmd=Retrieve $\& \mathrm{db}=$ PubMed\&list_uids $=12747876 \& d o p t=$ Abstract

Loveman E, Royle P, Waugh N. Specialist nurses in diabetes mellitus. Cochrane Database Syst Rev 2003;(2):CD003286. http:/ / www.ncbi.nlm.nih.gov/entrez/query.fcgi?cmd=Retrieve $\& d b=$ PubMed\&list_uids=12804458\&dopt=Abstract

Luckey MM, Gilchrist N, Bone HG, et al. Therapeutic equivalence of alendronate 35 milligrams once weekly and 5 milligrams daily in the prevention of postmenopausal osteoporosis. Obstet Gynecol 2003;101:711-21. http://www.ncbi.nlm. nih.gov/entrez/query.fcgi?cmd=Retrieve\&db=PubMed\&list_uids $=12681875 \&$ dopt $=$ Abstract

MacGillivray S, Arroll B, Hatcher S, et al. Efficacy and tolerability of selective serotonin reuptake inhibitors compared with tricyclic antidepressants in depression treated in primary care: systematic review and meta-analysis. BMJ 2003;326:1014-17. http://www.ncbi.nlm.nih.gov/entrez/query.fcgi?cmd=Retrieve $\& \mathrm{db}=$ PubMed\&list_uids=12742924\&dopt=Abstract

Marjoribanks J, Lethaby A, Farquhar C. Surgery versus medical therapy for heavy menstrual bleeding. Cochrane Database Syst Rev 2003;(2):CD003855. http://www.ncbi.nlm.nih.gov/entrez/ query.fcgi? $\mathrm{cmd}=$ Retrieve $\& \mathrm{db}=$ PubMed\&list_uids $=12804492 \&$ $\mathrm{dopt}=\mathrm{Abstract}$ 
Massaga JJ, Kitua AY, Lemnge MM, et al. Effect of intermittent treatment with amodiaquine on anaemia and malarial fevers in infants in Tanzania: a randomised placebo-controlled trial. Lancet 2003;361:1853-60. http://www.ncbi.nlm.nih.gov/entrez/ query.fcgi? $\mathrm{cmd}=$ Retrieve $\& \mathrm{db}=$ PubMed\&list_uids $=12788572 \&$ $\mathrm{dop}=\mathrm{Abstract}$

McCrory DC, Williams JW, Dolor RJ, et al. Management of allergic rhinitis in the working-age population. Evidence Report/ Technology Assessment Number 67. AHRQ Publication No. 03-E015. Rockville, MD: Agency for Healthcare Research and Quality, Mar 2003. http://www.ahrq.gov/clinic/epcsums/ rhinworksum.htm

McErlean M, Bartfield JM, Karunakar TA, et al. Midazolam syrup as a premedication to reduce the discomfort associated with pediatric intravenous catheter insertion.J Pediatr 2003;142:429-30. http://www.ncbi.nlm.nih.gov/entrez/query.fcgi?cmd=Retrieve $\& d b=$ PubMed\&list_uids=12712062\&dopt=Abstract

Meigs JB, Cagliero E, Dubey A, et al. A controlled trial of web-based diabetes disease management: the MGH Diabetes Primary Care Improvement Project. Diabetes Care 2003;26:750-7. http://www.ncbi.nlm.nih.gov/entrez/query.fcgi?cmd=Retrieve $\& \mathrm{db}=$ PubMed\&list_uids=12610033\&dopt=Abstract

Meremikwu M, Oyo-Ita A. Physical methods for treating fever in children. Cochrane Database Syst Rev 2003;(2):CD004264. http:// www.ncbi.nlm.nih.gov/entrez/query.fcgi? cmd=Retrieve\&db= PubMed\&list_uids=12804512\&dopt=Abstract

Mermelstein R, Hedeker D, Wong SC. Extended telephone counseling for smoking cessation: does content matter? J Consult Clin Psychol 2003;71:565-74. http://www.ncbi.nlm.nih.gov/entrez/ query.fcgi? $\mathrm{cmd}=$ Retrieve\&db=PubMed\&list_uids=12795579 \&dopt=Abstract

Miller L, Hughes JP. Continuous combination oral contraceptive pills to eliminate withdrawal bleeding: a randomized trial. Obstet Gynecol 2003;101:653-61. http://www.ncbi.nlm.nih.gov/entrez/ query.fcgi? $\mathrm{cmd}=$ Retrieve $\& \mathrm{db}=\mathrm{PubMed} \&$ list_uids $=12681866 \&$ $\mathrm{dopt}=$ Abstract

Milne S, Brosseau L, Robinson V, et al. Continuous passive motion following total knee arthroscopy. Cochrane Database Syst Rev 2003;(2):CD004260. http://www.ncbi.nlm.nih.gov/entrez/ query.fcgi?cmd=Retrieve\&db=PubMed\&list_uids $=12804511 \&$ $\mathrm{dop}=$ Abstract

Mion LC, Palmer RM, Meldon SW, et al. Case finding and referral model for emergency department elders: a randomized clinical trial. Ann Emerg Med 2003;41:57-68. http://www.ncbi.nlm. nih.gov/entrez/query.fcgi? $c m d=R e t r i e v e \& d b=P u b M e d \& l i s t \_u i d s=$ $12514683 \&$ dopt=Abstract

Moher M, Hey K, Lancaster T. Workplace interventions for smoking cessation. Cochrane Database Syst Rev 2003;(2):CD003440. http://www.ncbi.nlm.nih.gov/entrez/query.fcgi?cmd=Retrieve $\& \mathrm{db}=$ PubMed\&list_uids $=12804467 \& d o p t=$ Abstract

Murray L, Cooper PJ, Wilson A, et al. Controlled trial of the short- and long-term effect of psychological treatment of postpartum depression: 2. Impact on the mother-child relationship and child outcome. Br J Psychiatry 2003;182:420-7. http:// www.ncbi.nlm.nih.gov/entrez/query.fcgi?cmd=Retrieve $\& \mathrm{db}=$ PubMed\&list_uids=12724245\&dopt=Abstract
Nixon RD, Sweeney L, Erickson DB, et al. Parent-child interaction therapy: a comparison of standard and abbreviated treatments for oppositional defiant preschoolers. J Consult Clin Psychol 2003;71:251-60. http://www.ncbi.nlm.nih.gov/entrez/ query.fcgi? $\mathrm{cmd}=$ Retrieve $\& \mathrm{db}=$ PubMed\&list_uids $=12699020 \&$ $\mathrm{dopt}=\mathrm{Abstract}$

O'Connor AM, Stacey D, Entwistle V, et al. Decision aids for people facing health treatment or screening decisions. Cochrane Database Syst Rev 2003;(2):CD001431. http://www.ncbi.nlm. nih.gov/entrez/query.fcgi? $c m d=$ Retrieve $\& \mathrm{db}=\mathrm{PubMed} \&$ list_uids $=$ $12804407 \&$ dopt $=$ Abstract

Patel SR, White DP, Malhotra A, et al. Continuous positive airway pressure therapy for treating sleepiness in a diverse population with obstructive sleep apnea: results of a meta-analysis. Arch Intern Med 2003;163:565-71.http://www.ncbi.nlm.nih.gov/entrez/ query.fcgi? $\mathrm{cmd}=$ Retrieve $\& \mathrm{db}=$ PubMed\&list_uids $=12622603 \&$ $\mathrm{dopt}=$ Abstract

Pauwels RA, Pedersen S, Busse WW, et al. Early intervention with budesonide in mild persistent asthma: a randomised, doubleblind trial. Lancet 2003;361:1071-6. http://www.ncbi.nlm.nih. gov/entrez/query.fcgi?cmd=Retrieve\&db=PubMed\&list_uids $=$ 12672309\&dopt=Abstract

Poole PJ, Black PN. Mucolytic agents for chronic bronchitis or chronic obstructive pulmonary disease. Cochrane Database Syst Rev 2003;(2):CD001287. http://www.ncbi.nlm.nih.gov/entrez/ query.fcgi? $\mathrm{cmd}=$ Retrieve\&db=PubMed\&list_uids=12804402\& dopt=Abstract

Powell H, Gibson PG. Inhaled corticosteroid doses in asthma: an evidence-based approach. Med J Aust 2003;178:223-5. http:// www.ncbi.nlm.nih.gov/entrez/query.fcgi? $\mathrm{cmd}=$ Retrieve\&db= PubMed\&list_uids=12603186\&dopt=Abstract

Psaty BM, Lumley T, Furberg CD, et al. Health outcomes associated with various antihypertensive therapies used as first-line agents: a network meta-analysis. JAMA 2003;289:2534-44. http://www.ncbi.nlm.nih.gov/entrez/query.fcgi?cmd=Retrieve $\& \mathrm{db}=$ PubMed\&list_uids $=12759325 \&$ dopt $=$ Abstract

Pwee KH, Shukla VK, Herrmann N, et al. Novel antipsychotics for agitation in dementia: a systematic review. Ottawa: Canadian Coordinating Office for Health Technology Assessment (CCOHTA), Mar 2003. www.ccohta.ca

Randolph AG, Wypij D, Venkataraman ST, et al. Effect of mechanical ventilator weaning protocols on respiratory outcomes in infants and children: a randomized controlled trial. JAMA 2002;288:2561-8. http://www.ncbi.nlm.nih.gov/entrez/ query.fcgi? $\mathrm{cmd}=$ Retrieve $\& \mathrm{db}=$ PubMed\&list_uids $=12444863 \&$ $\mathrm{dopt}=\mathrm{Abstract}$

Rea MM, Tompson MC, Miklowitz DJ, et al. Family-focused treatment versus individual treatment for bipolar disorder: results of a randomized clinical trial. J Consult Clin Psychol 2003;71:482-92. http://www.ncbi.nlm.nih.gov/entrez/query. fcgi? cmd=Retrieve\&db=PubMed\&list_uids $=12795572 \& d o p t=$ Abstract

Riemsma RP, Kirwan JR, Taal E, et al. Patient education for adults with rheumatoid arthritis. Cochrane Database Syst Rev 2003;(2):CD003688. http://www.ncbi.nlm.nih.gov/entrez/ query.fcgi? $\mathrm{cmd}=$ Retrieve $\& \mathrm{db}=$ PubMed\&list_uids $=12804484 \&$ $\mathrm{dopt}=$ Abstract 
Rodríguez-Morán M, Guerrero-Romero F. Oral magnesium supplementation improves insulin sensitivity and metabolic control in type 2 diabetic subjects: a randomized double-blind controlled trial. Diabetes Care 2003;26:1147-52. http://www.ncbi. nlm.nih.gov/entrez/query.fcgi? $\mathrm{cmd}=$ Retrieve $\& \mathrm{db}=$ PubMed\& list_uids $=12663588 \& d o p t=A b s t r a c t$

Salpeter S, Greyber E, Pasternak G, et al. Risk of fatal and nonfatal lactic acidosis with metformin use in type 2 diabetes mellitus. Cochrane Database Syst Rev 2003;(2):CD002967. http:// www.ncbi.nlm.nih.gov/entrez/query.fcgi? $c m d=$ Retrieve $\& \mathrm{db}=$ PubMed\&list_uids=12804446\&dopt=Abstract

Sandler IN, Ayers TS, Wolchik SA, et al. The Family Bereavement Program: efficacy evaluation of a theory-based prevention program for parentally bereaved children and adolescents. J Consult Clin Psychol 2003;71:587-600. http:// www.ncbi.nlm.nih.gov/entrez/query.fcgi? $\mathrm{cmd}=$ Retrieve\&db= PubMed\&list_uids $=12795581 \&$ dopt=Abstract

Schnurr PP, Friedman MJ, Foy DW, et al. Randomized trial of trauma-focused group therapy for posttraumatic stress disorder: results from a Department of Veterans Affairs cooperative study. Arch Gen Psychiatry 2003;60:481-9. http://www.ncbi.nlm. nih.gov/entrez/query.fcgi? $c$ cmd=Retrieve\&db=PubMed\&list_uids $=12742869 \&$ dopt $=$ Abstract

Segal JB, Eng J, Jenckes MW, et al. Diagnosis and treatment of deep venous thrombosis and pulmonary embolism. Evidence Report/ Technology Assessment Number 68. AHRQ Publication No. 03-E016. Rockville, MD: Agency for Healthcare Research and Quality, Mar 2003. http://www.ahcpr.gov/clinic/epcsums/ dvtsum.htm

Sheikh A, Hurwitz B. House dust mite avoidance measures for perennial allergic rhinitis: a systematic review of efficacy. $\mathrm{Br} J$ Gen Pract 2003;53:318-22.

Scott JT, Harmsen M, Prictor MJ, et al. Recordings or summaries of consultations for people with cancer. Cochrane Database Syst Rev 2003;(2):CD001539. http://www.ncbi.nlm.nih.gov/entrez/ query.fcgi? $\mathrm{cmd}=$ Retrieve $\& \mathrm{db}=$ PubMed\&list_uids $=12804411 \&$ dopt=Abstract

Sharpe L, Sensky T, Timberlake N, et al. Long-term efficacy of a cognitive behavioural treatment from a randomized controlled trial for patients recently diagnosed with rheumatoid arthritis. Rheumatology 2003;42:435-41. http://www.ncbi.nlm.nih.gov/entrez/ query.fcgi? $\mathrm{cmd}=$ Retrieve\&db=PubMed\&list_uids $=12626793 \&$ dopt=Abstract

Shekelle PG, Hardy ML, Morton SC, et al. Efficacy and safety of ephedra and ephedrine for weight loss and athletic performance: a meta-analysis. JAMA 2003;289:1537-45. http://www.ncbi. nlm.nih.gov/entrez/query.fcgi?cmd=Retrieve\&db=PubMed\& list_uids=12672771\&dopt=Abstract

Stearns V, Beebe KL, Iyengar M, et al. Paroxetine controlled release in the treatment of menopausal hot flashes: a randomized controlled trial. JAMA 2003;289:2827-34. http:// www.ncbi.nlm.nih.gov/entrez/query.fcgi? $\mathrm{cmd}=$ Retrieve\&db= PubMed\&list uids $=12783913 \&$ dopt $=$ Abstract

Steptoe A, Perkins-Porras L, McKay C, et al. Behavioural counselling to increase consumption of fruit and vegetables in low income adults: randomised trial. BMJ 2003;326:855-60.
http://www.ncbi.nlm.nih.gov/entrez/query.fcgi?cmd=Retrieve $\& \mathrm{db}=$ PubMed\&list_uids=12702620\&dopt=Abstract

Steultjens EM, Dekker J, Bouter LM, et al. Occupational therapy for stroke patients: a systematic review. Stroke 2003;34:676-87. http://www.ncbi.nlm.nih.gov/entrez/query.fcgi?cmd=Retrieve $\& d b=$ PubMed\&list_uids=12624291\&dopt=Abstract

Tate DF, Jackvony EH, Wing RR. Effects of Internet behavioral counseling on weight loss in adults at risk for type 2 diabetes: a randomized trial. JAMA 2003;289:1833-6. http://www.ncbi.nlm. nih.gov/entrez/query.fcgi? $c$ de=Retrieve\&db=PubMed\&list_uids $=12684363 \&$ dopt $=$ Abstract

Taylor KL, Lamdan RM, Siegel JE, et al. Psychological adjustment among African American breast cancer patients: one-year follow-up results of a randomized psychoeducational group intervention. Health Psychol 2003;22:316-23. http:// www.ncbi.nlm.nih.gov/entrez/query.fcgi? $\mathrm{cmd}=$ Retrieve\&db= PubMed\&list_uids=12790260\&dopt=Abstract

Tesch BJ. Herbs commonly used by women: an evidence-based review. Am J Obstet Gynecol 2003;188:S44-55. http://www.ncbi. nlm.nih.gov/entrez/query.fcgi? $c$ md=Retrieve\&db=PubMed $\&$ list_uids=12748451\&dopt=Abstract

Towheed TE, Judd MJ, Hochberg MC, et al. Acetaminophen for osteoarthritis. Cochrane Database Syst Rev 2003;(2);CD004257. http://www.ncbi.nlm.nih.gov/entrez/query.fcgi? cmd=Retrieve $\& \mathrm{db}=$ PubMed\&list_uids $=12804508 \& d o p t=$ Abstract

Tsay SL, Rong JR, Lin PF. Acupoints massage in improving the quality of sleep and quality of life in patients with end-stage renal disease. J Adv Nurs 2003;42:134-42. http:// www.ncbi.nlm.nih.gov/entrez/query.fcgi? cmd=Retrieve $\& \mathrm{db}=$ PubMed\&list uids=12670382\&dopt=Abstract

van de Wetering MD, van Woensel JBM. Prophylactic antibiotics for preventing early central venous catheter gram positive infections in oncology patients. Cochrane Database Syst Rev 2003;(2); CD003295. http://www.ncbi.nlm.nih.gov/entrez/query.fcgi? cmd=Retrieve $\& d b=$ PubMed\&list_uids=12804460\&dopt=Abstract

van der Meer RM, Wagena EJ, Ostelo RWJG, et al. Smoking cessation for chronic obstructive pulmonary disease. Cochrane Database Syst Rev 2003;(2):CD002999. http://www.ncbi.nlm.nih. gov/entrez/query.fcgi? $c m d=$ Retrieve\&db=PubMed\&list_uids= $12804448 \&$ dopt=Abstract

van Schoor NM, Smit JH, Twisk JW, et al. Prevention of hip fractures by external hip protectors: a randomized controlled trial. JAMA 2003;289:1957-62. http://www.ncbi.nlm.nih.gov/entrez/ query.fcgi? $\mathrm{cmd}=$ Retrieve $\& \mathrm{db}=$ PubMed\&list_uids $=12697798 \&$ $\mathrm{dopt}=\mathrm{Abstract}$

Walsh JM, Terdiman JP. Colorectal cancer screening: scientific review.JAMA 2003;289:1288-96. http://www.ncbi.nlm.nih.gov/ entrez/query.fcgi?cmd=Retrieve\&db=PubMed\&list_uids= 12633191\&dopt=Abstract

Walters EH, Walters J. Inhaled short acting beta2-agonist use in chronic asthma: regular versus as needed treatment. Cochrane Database Syst Rev 2003;(2):CD001285. http://www.ncbi.nlm. nih.gov/entrez/query.fcgi?cmd=Retrieve $\& \mathrm{db}=$ PubMed\&list_uids $=12804401 \& d o p t=$ Abstract 
Weaver CS, Terrell KM. Evidence-based emergency medicine. Update: do ophthalmic nonsteroidal anti-inflammatory drugs reduce the pain associated with simple corneal abrasion without delaying healing? Ann Emerg Med 2003;41:134-40. http:// www.ncbi.nlm.nih.gov/entrez/query.fcgi? $\mathrm{cmd}=$ Retrieve\&db= PubMed\&list_uids=12514694\&dopt=Abstract

Weiss B, Harris V, Catron T, et al. Efficacy of the RECAP intervention program for children with concurrent internalizing and externalizing problems. J Consult Clin Psychol 2003;71:364-74. http://www.ncbi.nlm.nih.gov/entrez/query. fcgi? cmd=Retrieve\&db=PubMed\&list_uids=12699030\&dopt= Abstract

Weissberg-Benchell J, Antisdel-Lomaglio J, Seshadri R. Insulin pump therapy: a meta-analysis. Diabetes Care 2003;26:1079-87. http://www.ncbi.nlm.nih.gov/entrez/query.fcgi? cmd=Retrieve $\& \mathrm{db}=$ PubMed\&list_uids=12663577\&dopt=Abstract

Wenzl TG, Schneider S, Scheele F, et al. Effects of thickened feeding on gastroesophageal reflux in infants: a placebocontrolled crossover study using intraluminal impedance. Pediatrics 2003;111:e355-9. http://www.ncbi.nlm.nih.gov/ entrez/query.fcgi?cmd=Retrieve\&db=PubMed\&list_uids= 12671151\&dopt=Abstract

Wolfe DA, Wekerle C, Scott K, et al. Dating violence prevention with at-risk youth: a controlled outcome evaluation. J Consult Clin Psychol 2003;71:279-91. http://www.ncbi.nlm.nih.gov/ entrez/query.fcgi?cmd=Retrieve $\& d b=$ PubMed\&list_uids $=$ $12699022 \&$ dopt $=$ Abstract

Wormser GP, Ramanathan R, Nowakowski J, et al. Duration of antibiotic therapy for early Lyme disease. A randomized, double-blind, placebo-controlled trial. Ann Intern Med 2003;138: 697-704.http://www.ncbi.nlm.nih.gov/entrez/query.fcgi?cmd= Retrieve\&db=PubMed\&list_uids=12729423\&dopt=Abstract

Wysocki T, Harris MA, Mauras N, et al. Absence of adverse effects of severe hypoglycemia on cognitive function in school-aged children with diabetes over 18 months. Diabetes Care 2003;26: 1100-5. http://www.ncbi.nlm.nih.gov/entrez/query.fcgi?cmd $=$ Retrieve $\& d b=$ PubMed\&list_uids $=12663580 \& d o p t=$ Abstract

Yeh GY, Eisenberg DM, Kaptchuk TJ, et al. Systematic review of herbs and dietary supplements for glycemic control in diabetes. Diabetes Care 2003;26:1277-94. http://www.ncbi.nlm.nih.gov/ entrez/query.fcgi?.cmd=Retrieve\&db=PubMed\&list_uids= 12663610\&dopt=Abstract

Ylinen J, Takala EP, Nykänen M, et al. Active neck muscle training in the treatment of chronic neck pain in women: a randomized controlled trial. JAMA 2003;289:2509-16. http:// www.ncbi.nlm.nih.gov/entrez/query.fcgi?cmd=Retrieve\&db= PubMed\&list_uids=12759322\&dopt=Abstract

Zorc JJ, Scarfone RJ, Li Y, et al. Scheduled follow-up after a pediatric emergency department visit for asthma: a randomized trial. Pediatrics 2003;111:495-502. http://www.ncbi.nlm.nih.gov/ entrez/query.fcgi? $c$ do=Retrieve\&db=PubMed\&list_uids= $12612227 \&$ dopt $=$ Abstract

\section{Assessment (screening and diagnosis)}

Anand SS, Razak F, Vuksan V, et al. Diagnostic strategies to detect glucose intolerance in a multiethnic population. Diabetes Care 2003;26:290-6. http://www.ncbi.nlm.nih.gov/entrez/ query.fcgi?cmd=Retrieve $\& d b=$ PubMed\&list_uids $=12547851 \&$ $\mathrm{dopt}=\mathrm{Abstract}$
Jean-Mary MB, Dicanzio J, Shaw J, et al. Limited accuracy and reliability of infrared axillary and aural thermometers in a pediatric outpatient population. J Pediatr 2002;141:671-6. http:// www.ncbi.nlm.nih.gov/entrez/query.fcgi? $\mathrm{cmd}=$ Retrieve $\& d b=$ PubMed\&list_uids=12410196\&dopt=Abstract

Lee DT, Yip AS, Chan SS, et al. Postdelivery screening for postpartum depression. Psychosom Med 2003;65:357-61. http:// www.ncbi.nlm.nih.gov/entrez/query.fcgi? $\mathrm{cmd}=$ Retrieve $\& d b=$ PubMed\&list_uids=12764207\&dopt=Abstract

Leibson CL, Tosteson AN, Gabriel SE, et al. Mortality, disability, and nursing home use for persons with and without hip fracture: a population-based study. $J$ Am Geriatr Soc 2002;50:1644-50. http://www.ncbi.nlm.nih.gov/entrez/query. fcgi? $c m d=$ Retrieve\&db=PubMed\&list_uids=12366617\&dopt= Abstract

Mourad O, Palda V, Detsky AS. A comprehensive evidencebased approach to fever of unknown origin. Arch Intern Med 2003;163:545-51. http://www.ncbi.nlm.nih.gov/entrez/query. fcgi? $\mathrm{cmd}=$ Retrieve\&db=PubMed\&list_uids=12622601\&dopt= Abstract

Rinaldi P, Mecocci P, Benedetti C, et al. Validation of the five-item Geriatric Depression Scale in elderly subjects in three different settings. J Am Geriatr Soc 2003;51:694-8. http://www.ncbi. nlm.nih.gov/entrez/query.fcgi?cmd=Retrieve $\& d b=$ PubMed\& list_uids=12752847\&dopt=Abstract

Shekelle P, Takata G, Chan LS. Diagnosis, natural history, and late effects of otitis media with effusion. Evidence Report/Technology Assessment Number 55. AHRQ Publication No. 03-E023. Rockville, MD: Agency for Healthcare Research and Quality, May 2003. http://www.ahrq.gov/clinic/epcsums/otdiagsum.htm

Yueh B, Shapiro N, MacLean CH, et al. Screening and management of adult hearing loss in primary care: scientific review. JAMA 2003;289:1976-85. http://www.ncbi.nlm.nih.gov/entrez/ query.fcgi? $c$ de=Retrieve $\& d b=$ PubMed\&list_uids $=12697801 \&$ dopt=Abstract

\section{Prognosis}

Aben I, Verhey F, Strik J, et al. A comparative study into the one year cumulative incidence of depression after stroke and myocardial infarction. J Neurol Neurosurg Psychiatry 2003;74: 581-5. http://www.ncbi.nlm.nih.gov/entrez/query.fcgi? $\mathrm{cmd}=$ Retrieve\&db=PubMed\&list_uids=12700297\&dopt=Abstract

Albareda M, Caballero A, Badell G, et al. Diabetes and abnormal glucose tolerance in women with previous gestational diabetes. Diabetes Care 2003;26:1199-205. http://www.ncbi.nlm.nih.gov/ entrez/query.fcgi?.cmd=Retrieve\&db=PubMed\&list_uids= $12663597 \&$ dopt=Abstract

Ardissino G, Daccò V, Testa S, et al. Epidemiology of chronic renal failure in children: data from the ItalKid project. Pediatrics 2003;111:e382-7. http://www.ncbi.nlm.nih.gov/entrez/query. fcgi? cmd=Retrieve\&db=PubMed\&list_uids=12671156\&dopt= Abstract

Booth CM, Matukas LM, Tomlinson GA, et al. Clinical features and short-term outcomes of 144 patients with SARS in the greater Toronto area. JAMA 2003;289:2801-9. http:// www.ncbi.nlm.nih.gov/entrez/query.fcgi?cmd=Retrieve $\& d b=$ PubMed\&list_uids=12734147\&dopt=Abstract 
Combes A, Figliolini C, Trouillet JL, et al. Factors predicting ventilator-associated pneumonia recurrence. Crit Care Med 2003;31:1102-7. http://www.ncbi.nlm.nih.gov/entrez/query. fcgi?cmd=Retrieve\&db=PubMed\&list_uids=12682479\&dopt= Abstract

Degner LF, Hack T, O'Neil J, et al. A new approach to eliciting meaning in the context of breast cancer. Cancer Nurs 2003;26:169-78. http://www.ncbi.nlm.nih.gov/entrez/query. fcgi? $c$ dendrieve\&db=PubMed\&list_uids=12832949\&dopt= Abstract

Eberly LE, Cohen JD, Prineas R, et al. Impact of incident diabetes and incident nonfatal cardiovascular disease on 18-year mortality: the Multiple Risk Factor Intervention trial experience. Diabetes Care 2003;26:848-54. http://www.ncbi.nlm.nih.gov/ entrez/query.fcgi?cmd=Retrieve\&db=PubMed\&list_uids= $12610048 \&$ dopt=Abstract

Howieson DB, Camicioli R, Quinn J, et al. Natural history of cognitive decline in the old old. Neurology 2003;60:1489-94. http://www.ncbi.nlm.nih.gov/entrez/query.fcgi?cmd= Retrieve\&db=PubMed\&list_uids=12743237\&dopt=Abstract

Korndörfer SR, Lucas AR, Suman VJ, et al. Long-term survival of patients with anorexia nervosa: a population-based study in Rochester, Minn. Mayo Clin Proc 2003;78:278-84. http:// www.ncbi.nlm.nih.gov/entrez/query.fcgi?cmd=Retrieve $\& \mathrm{db}=$ PubMed\&list_uids $=12630579 \& d o p t=A b s t r a c t$

Solomon DH, Karlson EW, Rimm EB, et al. Cardiovascular morbidity and mortality in women diagnosed with rheumatoid arthritis. Circulation 2003;107:1303-7. http://www.ncbi.nlm. nih.gov/entrez/

query.fcgi? $\mathrm{cmd}=$ Retrieve $\& \mathrm{db}=$ PubMed\&list_uids $=$ $12628952 \&$ dopt $=$ Abstract

Taylor MD, Frier BM, Gold AE, et al. Psychosocial factors and diabetes-related outcomes following diagnosis of Type 1 diabetes in adults: the Edinburgh Prospective Diabetes Study. Diabet Med 2003;20:135-46. http://www.ncbi.nlm.nih.gov/ entrez/query.fcgi?cmd=Retrieve\&db=PubMed\&list_uids= $12581265 \&$ dopt=Abstract

\section{Causation}

Alavanja MC, Samanic C, Dosemeci M, et al. Use of agricultural pesticides and prostate cancer risk in the Agricultural Health Study cohort. Am J Epidemiol 2003;157:800-14. http:// www.ncbi.nlm.nih.gov/entrez/query.fcgi?cmd=Retrieve $\& \mathrm{db}=$ PubMed\&list_uids=12727674\&dopt=Abstract

Barnes LL, Wilson RS, Schneider JA, et al. Gender, cognitive decline, and risk of $\mathrm{AD}$ in older persons. Neurology 2003;60:1777-81. http://www.ncbi.nlm.nih.gov/entrez/query. fcgi?cmd=Retrieve $\& d b=$ PubMed\&list_uids=12796530\&dopt= Abstract

Casper RC, Fleisher BE, Lee-Ancajas JC, et al. Follow-up of children of depressed mothers exposed or not exposed to antidepressant drugs during pregnancy.J Pediatr 2003;142:402-8. http://www.ncbi.nlm.nih.gov/entrez/query.fcgi?cmd=Retrieve $\& \mathrm{db}=$ PubMed\&list_uids=12712058\&dopt=Abstract
Cole C, Binney G, Casey P, et al. Criteria for determining disability in infants and children: low birth weight. Evidence Report/ Technology Assessment Number 70. AHRQ Publication No. 03-E010. Rockville, MD: Agency for Healthcare Research and Quality, Dec 2002. http://www.ahrq.gov/clinic/tp/lbwdistp.htm

Cole MG, Dendukuri N. Risk factors for depression among elderly community subjects: a systematic review and meta-analysis. Am J Psychiatry 2003;160:1147-56. http://www.ncbi.nlm.nih. gov/entrez/query.fcgi?cmd=Retrieve $\& \mathrm{db}=$ PubMed\&list_uids= $12777274 \&$ dopt=Abstract

Critchley JA, Unal B. Health effects associated with smokeless tobacco: a systematic review. Thorax 2003;58:435-43. http:// www.ncbi.nlm.nih.gov/entrez/query.fcgi?cmd=Retrieve $\& \mathrm{db}=$ PubMed\&list_uids=12728167\&dopt=Abstract

de Beer BA, Graamans K, Snik AF, et al. Hearing deficits in young adults who had a history of otitis media in childhood: use of personal stereos had no effect on hearing. Pediatrics 2003;111:e304-8. http://www.ncbi.nlm.nih.gov/entrez/query. fcgi? cmd=Retrieve\&db=PubMed\&list_uids=12671143\&dopt= Abstract

Dey DK, Rothenberg E, Sundh V, et al. Waist circumference, body mass index, and risk for stroke in older people: a 15 year longitudinal population study of 70-year-olds. J Am Geriatr Soc 2002;50:1510-8. http://www.ncbi.nlm.nih.gov/entrez/query. fcgi? $c m d=$ Retrieve\&db=PubMed\&list_uids=12383148\&dopt= Abstract

Engeland A, Bjørge T, Søgaard AJ, et al. Body mass index in adolescence in relation to total mortality: 32-year follow-up of 227,000 Norwegian boys and girls. Am J Epidemiol 2003;157:517-23. http://www.ncbi.nlm.nih.gov/entrez/query. fcgi? cmd=Retrieve\&db=PubMed\&list_uids=12631541\&dopt= Abstract

Franks P, Gold MR, Fiscella K. Sociodemographics, self-rated health, and mortality in the US. Soc Sci Med 2003;56:2505-14. http://www.ncbi.nlm.nih.gov/entrez/query.fcgi?cmd=Retrieve $\& \mathrm{db}=$ PubMed\&list_uids=12742613\&dopt=Abstract

Hopfer CJ, Crowley TJ, Hewitt JK. Review of twin and adoption studies of adolescent substance use. J Am Acad Child Adolesc Psychiatry 2003;42:710-9.

Hu FB, Cho E, Rexrode KM, et al. Fish and long-chain omega-3 fatty acid intake and risk of coronary heart disease and total mortality in diabetic women. Circulation 2003;107:1852-7. http://www.ncbi.nlm.nih.gov/entrez/query.fcgi?cmd=Retrieve $\& d b=$ PubMed\&list_uids=12668520\&dopt=Abstract

Jacobsen BK, Heuch I, Kvåle G. Age at natural menopause and all-cause mortality: a 37-year follow-up of 19,731 Norwegian women. Am J Epidemiol 2003;157:923-9. http:// www.ncbi.nlm.nih.gov / entrez / query.fcgi? $\mathrm{cmd}=$ Retrieve\&db= PubMed\&list_uids=12746245\&dopt=Abstract

Kendler KS, Jacobson KC, Prescott CA, et al. Specificity of genetic and environmental risk factors for use and abuse/ dependence of cannabis, cocaine, hallucinogens, sedatives, stimulants, and opiates in male twins. Am J Psychiatry 2003;160:687-95. http://www.ncbi.nlm.nih.gov/entrez/query. fcgi?cmd=Retrieve\&db=PubMed\&list_uids=12668357\&dopt= Abstract 
Mannino DM, Buist AS, Petty TL, et al. Lung function and mortality in the United States: data from the First National Health and Nutrition Examination Survey follow up study. Thorax 2003;58:388-93. http://www.ncbi.nlm.nih.gov/entrez/query. fcgi? $\mathrm{cmd}=$ Retrieve $\& \mathrm{db}=$ PubMed\&list_uids $=12728157 \& d o p t=$ Abstract

Osler M, Andreasen AH, Hoidrup S. No inverse association between fish consumption and risk of death from all-causes, and incidence of coronary heart disease in middle-aged, Danish adults. J Clin Epidemiol 2003;56:274-9. http://www.ncbi.nlm. nih.gov/entrez/query.fcgi?cmd=Retrieve\&db=PubMed\&list_uids $=12725883 \& \mathrm{dopt}=\mathrm{Abstract}$

Owen CG, Whincup PH, Odoki K, et al. Birth weight and blood cholesterol level: a study in adolescents and systematic review. Pediatrics 2003;111:1081-9. http://www.ncbi.nlm.nih.gov/ entrez/query.fcgi? $\mathrm{cmd}=$ Retrieve $\& d b=$ PubMed\&list_uids $=$ $12728092 \&$ dopt $=$ Abstract

Pedersen A, Johansen C, Grønbaek M. Relations between amount and type of alcohol and colon and rectal cancer in a Danish population based cohort study. Gut 2003;52:861-7. http://www.ncbi.nlm.nih.gov/entrez/query.fcgi?cmd=Retrieve $\& \mathrm{db}=$ PubMed\&list_uids=12740343\&dopt=Abstract

Pharoah PO, Stevenson CJ, West CR. General Certificate of Secondary Education performance in very low birthweight infants. Arch Dis Child 2003;88:295-8. http://www.ncbi.nlm.nih.gov/ entrez/query.fcgi? $c$ d $=$ Retrieve\&db=PubMed\&list_uids= 12651749\&dopt=Abstract

Qin P, Agerbo E, Mortensen PB. Suicide risk in relation to socioeconomic, demographic, psychiatric, and familial factors: a national register-based study of all suicides in Denmark, 19811997. Am J Psychiatry 2003;160:765-72. http://www.ncbi.nlm.nih.

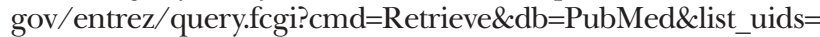
$12668367 \&$ dopt=Abstract

Smith JS, Green J, Berrington de Gonzalez A, et al. Cervical cancer and use of hormonal contraceptives: a systematic review. Lancet 2003;361:1159-67. http://www.ncbi.nlm.nih.gov/entrez/ query.fcgi? $c$ cmd=Retrieve $\& d b=$ PubMed\&list_uids=12686037 \&dopt=Abstract

Westman J, Hasselstrom J, Johansson SE, et al. The influences of place of birth and socioeconomic factors on attempted suicide in a defined population of 4.5 million people. Arch Gen Psychiatry 2003;60:409-14. http://www.ncbi.nlm.nih.gov/entrez/ query.fcgi? $\mathrm{cmd}=$ Retrieve $\& \mathrm{db}=$ PubMed\&list_uids $=12695319 \&$ $\mathrm{dopt}=\mathrm{Abstract}$

Wilson RS, Beckett LA, Bienias JL, et al. Terminal decline in cognitive function. Neurology 2003;60:1782-7. http://www.ncbi. nlm.nih.gov/entrez/query.fcgi? $\mathrm{cmd}=$ Retrieve $\& \mathrm{db}=$ PubMed\& list_uids=12796531\&dopt=Abstract

Wulsin LR, Singal BM. Do depressive symptoms increase the risk for the onset of coronary disease? A systematic quantitative review. Psychosom Med 2003;65:201-10. http://www.ncbi.nlm. nih.gov/entrez/

query.fcgi? cmd=Retrieve $\& d b=$ PubMed\&list_uids $=12651987 \&$ dopt=Abstract

Zhou W, Olsen J. Are complications after an induced abortion associated with reproductive failures in a subsequent pregnancy? Acta Obstet Gynecol Scand 2003;82:177-81. http://
www.ncbi.nlm.nih.gov/entrez/query.fcgi?cmd=Retrieve\& $\mathrm{db}=$ PubMed\&list_uids=12648182\&dopt=Abstract

\section{Quality improvement}

Bennett JW, Glasziou PP. Computerised reminders and feedback in medication management: a systematic review of randomised controlled trials. Med J Aust 2003;178:217-22. http://www.ncbi.nlm.nih.gov/entrez/query.fcgi?cmd=Retrieve $\& \mathrm{db}=$ PubMed\&list_uids=12603185\&dopt=Abstract

Brown AF, Mangione CM, Saliba D, et al. Guidelines for improving the care of the older person with diabetes mellitus. $J$ Am Geriatr Soc 2003;51:S265-80. http://www.ncbi.nlm.nih.gov/ entrez/query.fcgi?cmd=Retrieve\&db=PubMed\&list_uids= $12694461 \&$ dopt=Abstract

Fellowes D, Wilkinson S, Moore P. Communication skills training for health care professionals working with cancer patients, their families and/or carers. Cochrane Database Syst Rev 2003;(2):CD003751. http://www.ncbi.nlm.nih.gov/entrez/query. fcgi?cmd=Retrieve\&db=PubMed\&list_uids=12804489\&dopt= Abstract

Lim WK, Lambert SF, Gray LC. Effectiveness of case management and post-acute services in older people after hospital discharge. Med J Aust 2003;178:262-6. http://www.ncbi.nlm. nih.gov/entrez/query.fcgi?cmd=Retrieve $\& \mathrm{db}=$ PubMed\&list_uids $=12633482 \&$ dopt $=$ Abstract

Meredith S, Feldman P, Frey D, et al. Improving medication use in newly admitted home healthcare patients: a randomized controlled trial.J Am Geriatr Soc 2002;50:1484-91. http://www.ncbi. nlm.nih.gov/entrez/query.fcgi?cmd=Retrieve\&db=PubMed\& list_uids=12383144\&dopt=Abstract

\section{Economics}

Korthals-de Bos IB, Hoving JL, van Tulder MW, et al. Cost effectiveness of physiotherapy, manual therapy, and general practitioner care for neck pain: economic evaluation alongside a randomised controlled trial. BMJ 2003;326:911-4. http:// www.ncbi.nlm.nih.gov/entrez/query.fcgi? $\mathrm{cmd}=$ Retrieve\&db= PubMed\&list_uids=12714472\&dopt=Abstract

Plant PK, Owen JL, Parrott S, et al. Cost effectiveness of ward based non-invasive ventilation for acute exacerbations of chronic obstructive pulmonary disease: economic analysis of randomised controlled trial. BMJ 2003;326:956-9. http:// www.ncbi.nlm.nih.gov/entrez/query.fcgi? $\mathrm{cmd}=$ Retrieve\&db= PubMed\&list_uids=12727767\&dopt=Abstract

Scott J, Palmer S, Paykel E, et al. Use of cognitive therapy for relapse prevention in chronic depression. Cost-effectiveness study. Br J Psychiatry 2003;182:221-7. http://www.ncbi.nlm. nih.gov/entrez/query.fcgi? $c$ cmd=Retrieve $\& \mathrm{db}=$ PubMed\&list_uids $=12611785 \& \mathrm{dopt}=\mathrm{Abstract}$

van den Hout WB, Tijhuis GJ, Hazes JM, et al. Cost effectiveness and cost utility analysis of multidisciplinary care in patients with rheumatoid arthritis: a randomised comparison of clinical nurse specialist care, inpatient team care, and day patient team care. Ann Rheum Dis 2003;62:308-15. http://www.ncbi.nlm.nih.gov/ entrez/query.fcgi? $c$ de $=$ Retrieve $\& d b=$ PubMed\&list_uids $=$ $12634227 \&$ dopt=Abstract 


\section{Clinical prediction guide}

Callaghan LA, Cartwright DW, O'Rourke P, et al. Infant to staff ratios and risk of mortality in very low birthweight infants. Arch Dis Child Fetal Neonatal Ed 2003;88:F94-7. http://www.ncbi.nlm. nih.gov/entrez/query.fcgi? $\mathrm{cmd}=$ Retrieve\&db=PubMed\&list_uids $=12598494 \&$ dopt $=$ Abstract

Croce MA, Tolley EA, Fabian TC. A formula for prediction of posttraumatic pneumonia based on early anatomic and physiologic parameters. J Trauma 2003;54:724-30. http:// www.ncbi.nlm.nih.gov/entrez/query.fcgi?cmd=Retrieve $\& \mathrm{db}=$ PubMed\&list_uids=12707535\&dopt=Abstract

Fortescue EB, Kahn K, Bates DW. Major adverse outcomes after percutaneous transluminal coronary angioplasty: a clinical prediction rule.JClin Epidemiol 2003;56:17-27.http://www.ncbi.nlm. nih.gov/entrez/query.fcgi? $c$ cmd=Retrieve\&db=PubMed\&list_uids $=12589866 \&$ dopt $=$ Abstract

Gray NS, Hill C, McGleish A, et al. Prediction of violence and self-harm in mentally disordered offenders: a prospective study of the efficacy of HCR-20, PCL-R, and psychiatric symptomatology.J Consult Clin Psychol 2003;71:443-51. http://www.ncbi.nlm. nih.gov/entrez/query.fcgi? $\mathrm{cmd}=$ Retrieve\&db=PubMed\&list_uids $=12795569 \&$ dopt $=$ Abstract

Healey C, Osler TM, Rogers FB, et al. Improving the Glasgow Coma Scale score: motor score alone is a better predictor. $J$ Trauma 2003;54:671-80. http://www.ncbi.nlm.nih.gov/entrez/ query.fcgi? $\mathrm{cmd}=$ Retrieve $\& d b=$ PubMed\&list_uids $=12707528 \&$ dopt=Abstract

McGinn TG, Deluca J, Ahlawat SK, et al. Validation and modification of streptococcal pharyngitis clinical prediction rules. Mayo Clin Proc 2003;78:289-93. http://www.ncbi.nlm.nih.gov/entrez/ query.fcgi? $\mathrm{cmd}=$ Retrieve\&db=PubMed\&list_uids=12630581\& $\mathrm{dopt}=\mathrm{Abstract}$

\section{Qualitative}

Aaronson LS, Pallikkathayil L, Crighton F. A qualitative investigation of fatigue among healthy working adults. West J Nurs Res 2003;25:419-33. http://www.ncbi.nlm.nih.gov/entrez/query. fcgi? cmd=Retrieve\&db=PubMed\&list_uids $=12790057 \&$ dopt $=$ Abstract

Anderson EH, Spencer MH. Cognitive representations of AIDS: a phenomenological study. Qual Health Res 2002;12:1338-52. http://www.ncbi.nlm.nih.gov/entrez/query.fcgi?cmd=Retrieve $\& \mathrm{db}=$ PubMed\&list_uids=12474907\&dopt=Abstract

Arman M, Rehnsfeldt A. The hidden suffering among breast cancer patients: a qualitative metasynthesis. Qual Health Res 2003;13:510-27. http://www.ncbi.nlm.nih.gov/entrez/query. fcgi? $c m d=$ Retrieve $\& d b=$ PubMed\&list_uids=12703413\&dopt= Abstract

Bancroft A, Wiltshire S, Parry O, et al. "It's like an addiction first thing...afterwards it's like a habit": daily smoking behaviour among people living in areas of deprivation. Soc Sci Med 2003;56:1261-7. http://www.ncbi.nlm.nih.gov/entrez/query. fcgi? $c$ d $=$ Retrieve\&db=PubMed\&list_uids=12600363\&dopt= Abstract

Barthelsson C, Lützén K, Anderberg B, et al. Patients' experiences of laparoscopic cholecystectomy in day surgery. $J$ Clin Nurs 2003;12:253-9. http://www.ncbi.nlm.nih.gov/entrez/ query.fcgi? $\mathrm{cmd}=$ Retrieve\&db=PubMed\&list_uids $=12603558$ \&dopt=Abstract

Beitz JM, Zuzelo PR. The lived experience of having a neobladder. West J Nurs Res 2003;25:294-316. http://www.ncbi.nlm.nih. gov/entrez/

query.fcgi? cmd=Retrieve $\&$ db=PubMed\&list_uids=12705113\& dopt=Abstract

Broström A, Strömberg A, Dahlström U, et al. Congestive heart failure, spouses' support and the couple's sleep situation: a critical incident technique analysis. J Clin Nurs 2003;12:223-33. http://www.ncbi.nlm.nih.gov/entrez/query.fcgi?cmd=Retrieve $\& d b=$ PubMed\&list_uids=12603554\&dopt=Abstract

Brown JB, Carroll J, Boon H, et al. Women's decision-making about their health care: views over the life cycle. Patient Educ Couns 2002;48:225-31. http://www.ncbi.nlm.nih.gov/entrez/ query.fcgi? $\mathrm{cmd}=$ Retrieve\&db=PubMed\&list_uids $=12477607 \&$ $\mathrm{dopt}=$ Abstract

Burton NW, Turrell G, Oldenburg B. Participation in recreational physical activity: why do socioeconomic groups differ? Health Educ Behav 2003;30:225-44. http://www.ncbi.nlm.nih. gov/entrez/query.fcgi? cmd=Retrieve\&db=PubMed\&list_uids= $12693525 \&$ dopt=Abstract

Clegg A. Older South Asian patient and carer perceptions of culturally sensitive care in a community hospital setting. J Clin Nurs 2003;12:283-90. http://www.ncbi.nlm.nih.gov/entrez/ query.fcgi? $\mathrm{cmd}=$ Retrieve $\& \mathrm{db}=$ PubMed\&list_uids $=12603562 \&$ $\mathrm{dopt}=\mathrm{Abstract}$

Coeling HV, Biordi DL, Theis SL. Negotiating dyadic identity between caregivers and care receivers. J Nurs Scholarsh 2003;35:21-5. http://www.ncbi.nlm.nih.gov/entrez/query.fcgi? $\mathrm{cmd}=$ Retrieve $\& \mathrm{db}=$ PubMed\&list_uids $=12701522 \& \mathrm{dopt}=$ Abstract

Cronin C. First-time mothers-identifying their needs, perceptions and experiences. J Clin Nurs 2003;12:260-7. http:// www.ncbi.nlm.nih.gov/entrez/query.fcgi?cmd=Retrieve\&db= PubMed\&list_uids=12603559\&dopt=Abstract

Dalton CC, Gottlieb LN. The concept of readiness to change.J Adv Nurs 2003;42:108-17. http://www.ncbi.nlm.nih.gov/entrez/ query.fcgi? $\mathrm{cmd}=$ Retrieve $\& \mathrm{db}=$ PubMed\&list_uids $=12670379 \&$ dopt=Abstract

Elmberger E, Bolund C, Lützén K. Men with cancer. Changes in attempts to master the self-image as a man and as a parent. Cancer Nurs 2002;25:477-85. http://www.ncbi.nlm.nih.gov/entrez/ query.fcgi? $\mathrm{cmd}=$ Retrieve $\& \mathrm{db}=$ PubMed\&list_uids $=12464840 \&$ $\mathrm{dopt}=\mathrm{Abstract}$

Everitt H, Kumar S, Little P. A qualitative study of patients' perceptions of acute infective conjunctivitis. Br J Gen Pract 2003;53:36-41. http://www.ncbi.nlm.nih.gov/entrez/query.fcgi $? \mathrm{cmd}=$ Retrieve $\& \mathrm{db}=$ PubMed\&list_uids $=12564275 \& \mathrm{dopt}=$ Abstract

Ford S, Schofield T, Hope T. What are the ingredients for a successful evidence-based patient choice consultation? A qualitative study. Soc Sci Med 2003;56:589-602. http://www.ncbi.nlm.nih. gov/entrez/query.fcgi? $\mathrm{cmd}=$ Retrieve $\& \mathrm{db}=$ PubMed\&list_uids= 12570976\&dopt=Abstract 
Free C, Lee RM, Ogden J. Young women's accounts of factors influencing their use and non-use of emergency contraception: in-depth interview study. BMJ 2002;325:1393-6. http:// www.ncbi.nlm.nih.gov/entrez/query.fcgi? $\mathrm{cmd}=$ Retrieve\&db= PubMed\&list_uids $=12480855 \&$ dopt $=$ Abstract

Gray DE. Gender and coping: the parents of children with high functioning autism. Soc Sci Med 2003;56:631-42. http:// www.ncbi.nlm.nih.gov/entrez/query.fcgi? $\mathrm{cmd}=$ Retrieve\&db= PubMed\&list_uids=12570979\&dopt=Abstract

Hardin PK. Social and cultural considerations in recovery from anorexia nervosa: a critical poststructuralist analysis. ANS Adv Nurs Sci 2003;26:5-16. http://www.ncbi.nlm.nih.gov/entrez/ query.fcgi? $\mathrm{cmd}=$ Retrieve $\& \mathrm{db}=$ PubMed\&list uids $=12611426 \&$ dopt=Abstract

Hedström M, Haglund K, Skolin I, et al. Distressing events for children and adolescents with cancer: child, parent, and nurse perceptions. J Pediatr Oncol Nurs 2003;20:120-32. http:// www.ncbi.nlm.nih.gov/entrez/query.fcgi?cmd=Retrieve\&db= PubMed\&list_uids=12776260\&dopt=Abstract

Hjelm K, Nyberg P, Apelqvist J. Gender influences beliefs about health and illness in diabetic subjects with severe foot lesions. $J$ Adv Nurs 2002;40:673-84. http://www.ncbi.nlm.nih.gov/ entrez/query.fcgi? $\mathrm{cmd}=$ Retrieve\&db=PubMed\&list_uids= $12473048 \&$ dopt=Abstract

Huizinga GA, van der Graaf WT, Visser A, et al. Psychosocial consequences for children of a parent with cancer: a pilot study. Cancer Nurs 2003;26:195-202. http://www.ncbi.nlm.nih. gov/entrez/query.fcgi? $\mathrm{cmd}=$ Retrieve $\& \mathrm{db}=$ PubMed\&list_uids $=$ $12832952 \&$ dopt $=$ Abstract

King G, Cathers T, Brown E, et al. Turning points and protective processes in the lives of people with chronic disabilities. Qual Health Res 2003;13:184-206. http://www.ncbi.nlm.nih.gov/ entrez/query.fcgi?cmd=Retrieve\&db=PubMed\&list_uids= $12643028 \&$ dopt=Abstract

Maliski SL, Heilemann MV, McCorkle R. From "death sentence" to "good cancer": couples' transformation of a prostate cancer diagnosis. Nurs Res 2002;51:391-7.http://www.ncbi.nlm.nih.gov/ entrez/query.fcgi?cmd=Retrieve\&db=PubMed\&list_uids $=$ $12464759 \&$ dopt=Abstract

Maliski SL, Sarna L, Evangelista L, et al. The aftermath of lung cancer: balancing the good and bad. Cancer Nurs 2003;26: 237-44. http://www.ncbi.nlm.nih.gov/entrez/query.fcgi?cmd= Retrieve\&db=PubMed\&list_uids $=12832957 \&$ dopt=Abstract

McFall SL, Hamm RM. Interpretation of prostate cancer screening events and outcomes: a focus group study. Patient Educ Couns 2003;49:207-18. http://www.ncbi.nlm.nih.gov/entrez/ query.fcgi? $\mathrm{cmd}=$ Retrieve\&db=PubMed\&list_uids=12642192\& dopt=Abstract

Murray J, Ashworth R, Forster A, et al. Developing a primary care-based stroke service: a review of the qualitative literature. $\mathrm{Br}$ J Gen Pract 2003;53:137-42. http://www.ncbi.nlm.nih.gov/ entrez/query.fcgi? $c$ cmd=Retrieve\&db=PubMed\&list_uids= 12817361\&dopt=Abstract

Nekhlyudov L, Ross-Degnan D, Fletcher SW. Beliefs and expectations of women under 50 years old regarding screening mammography. J Gen Intern Med 2003;18:182-9. http://www.ncbi.
nlm.nih.gov/entrez/query.fcgi?cmd=Retrieve\&db=PubMed\& list_uids $=12648249 \&$ \&dopt $=$ Abstract

Nydén K, Petersson M, Nyström M. Unsatisfied basic needs of older patients in emergency care environments-obstacles to an active role in decision making. J Clin Nurs 2003;12:268-74. http://www.ncbi.nlm.nih.gov/entrez/query.fcgi?cmd=Retrieve\& $\mathrm{db}=$ PubMed\&list_uids=12603560\&dopt=Abstract

Ohman M, Söderberg S, Lundman B. Hovering between suffering and enduring: the meaning of living with serious chronic illness. Qual Health Res 2003;13:528-42. http://www.ncbi.nlm.nih. gov/entrez/query.fcgi?cmd=Retrieve $\& \mathrm{db}=$ PubMed\&list_uids= $12703414 \&$ dopt=Abstract

Paulson M, Norberg A, Söderberg S. Living in the shadow of fibromyalgic pain: the meaning of female partners' experiences. J Clin Nurs 2003;12:235-43. http://www.ncbi.nlm.nih.gov/

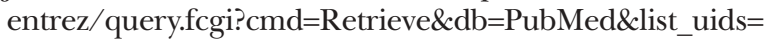
$12603556 \& d o p t=A b s t r a c t$

Polaschek N. Living on dialysis: concerns of clients in a renal setting. J Adv Nurs 2003;41:44-52. http://www.ncbi.nlm.nih. gov/entrez/query.fcgi? $\mathrm{cmd}=$ Retrieve $\& \mathrm{db}=$ PubMed\&list_uids $=$ $12519287 \&$ dopt $=$ Abstract

Prior L, Wood F, Lewis G, et al. Stigma revisited, disclosure of emotional problems in primary care consultations in Wales. Soc Sci Med 2003;56:2191-200. http://www.ncbi.nlm.nih.gov/ entrez/query.fcgi?cmd=Retrieve\&db=PubMed\&list_uids= $12697207 \&$ dopt $=$ Abstract

Rew L, Horner SD. Personal strengths of homeless adolescents living in a high-risk environment. ANS Adv Nurs Sci 2003;26:90101. http://www.ncbi.nlm.nih.gov/entrez/query.fcgi?.cmd= Retrieve\&db=PubMed\&list_uids=12795538\&dopt=Abstract

Roberts S, Livingston P, White V, et al. External breast prosthesis use: experiences and views of women with breast cancer, breast care nurses, and prosthesis fitters. Cancer Nurs 2003;26:179-86. http://www.ncbi.nlm.nih.gov/entrez/query.fcgi?cmd=Retrieve $\& d b=$ PubMed\&list_uids=12832950\&dopt=Abstract

Ryan S, Hassell A, Dawes P, et al. Control perceptions in patients with rheumatoid arthritis: the impact of the medical consultation. Rheumatology (Oxford) 2003;42:135-40. http://www.ncbi. nlm.nih.gov/entrez/query.fcgi? $c$ de $=$ Retrieve $\& d b=P u b M e d \&$ list_uids=12509626\&dopt=Abstract

Schoenberg NE, Peters JC, Drew EM. Unraveling the mysteries of timing: women's perceptions about time to treatment for cardiac symptoms. Soc Sci Med 2003;56:271-84. http:// www.ncbi.nlm.nih.gov/entrez/query.fcgi? $\mathrm{cmd}=$ Retrieve\&db= PubMed\&list_uids=12473313\&dopt=Abstract

Söderberg S, Strand M, Haapala M, et al. Living with a woman with fibromyalgia from the perspective of the husband. $J$ Adv Nurs 2003;42:143-50. http://www.ncbi.nlm.nih.gov/entrez/ query.fcgi? $\mathrm{cmd}=$ Retrieve $\& \mathrm{db}=$ PubMed\&list_uids $=12670383 \&$ dopt=Abstract

Swenson MM, MacLeod JS, Williams SD, et al. Quality of life after among ovarian germ cell cancer survivors: a narrative analysis. Oncol Nurs Forum 2003;30:380. http://www.ncbi. nlm.nih.gov/entrez/query.fcgi? $\mathrm{cmd}=$ Retrieve\&db=PubMed\& list_uids=12719752\&dopt=Abstract 
Tang ST. When death is imminent: where terminally ill patients with cancer prefer to die and why. Cancer Nurs 2003;26:245-51. http://www.ncbi.nlm.nih.gov/entrez/query.fcgi?.cmd=Retrieve $\& \mathrm{db}=$ PubMed\&list_uids=12832958\&dopt=Abstract

Thomé B, Dykes AK, Gunnars B, et al. The experiences of older people living with cancer. Cancer Nurs 2003;26:85-96. http:// www.ncbi.nlm.nih.gov/entrez/query.fcgi?cmd=Retrieve $\& \mathrm{db}=$ PubMed\&list_uids $=12660557 \& \mathrm{dopt}=$ Abstract

Thompson AM, Humbert ML, Mirwald RL. A longitudinal study of the impact of childhood and adolescent physical activity experiences on adult physical activity perceptions and behaviors. Qual Health Res 2003;13:358-77. http://www.ncbi.nlm.nih. gov/entrez/query.fcgi?cmd=Retrieve\&db=PubMed\&list_uids= $12669337 \& d o p t=A b s t r a c t$

Ward L, Innes M. Electronic medical summaries in general practice-considering the patient's contribution. BrJ Gen Pract 2003;53:293-7.

Widäng I, Fridlund B. Self-respect, dignity and confidence: conceptions of integrity among male patients. J Adv Nurs 2003; 42:47-56. http://www.ncbi.nlm.nih.gov/entrez/query.fcgi?cmd= Retrieve\&db=PubMed\&list_uids=12641811\&dopt=Abstract

Wilkes LM, O'Baugh J, Luke S, et al. Positive attitude in cancer: patients' perspectives. Oncol Nurs Forum 2003;30:412-6. http:// www.ncbi.nlm.nih.gov/entrez/query.fcgi? $c m d=$ Retrieve\&db= PubMed\&list_uids=12719741\&dopt=Abstract

Woodgate RL, Degner LF. A substantive theory of keeping the spirit alive: the spirit within children with cancer and their families. J Pediatr Oncol Nurs 2003;20:103-19. http://www.ncbi. nlm.nih.gov/entrez/query.fcgi? $\mathrm{cmd}=$ Retrieve $\& \mathrm{db}=$ PubMed\& list_uids $=127762598$ \&dopt $=$ Abstract

Worthington C, Myers T. Factors underlying anxiety in HIV testing: risk perceptions, stigma, and the patient-provider power dynamic. Qual Health Res 2003;13:636-55. http://www.ncbi.nlm. nih.gov/entrez/query.fcgi?cmd=Retrieve $\& d b=P u b M e d \&$ clist_uids $=12756685 \& \mathrm{dopt}=$ Abstract

Wuest J, Ford-Gilboe M, Merritt-Gray M, et al. Intrusion: the central problem for family health promotion among children and single mothers after leaving an abusive partner. Qual Health Res 2003;13:597-622. http://www.ncbi.nlm.nih.gov/entrez/ query.fcgi? $\mathrm{cmd}=$ Retrieve\&db=PubMed\&list_uids $=12756683 \&$ $\mathrm{dopt}=$ Abstract

Yi M. Decision-making process for living kidney donors. J Nurs Scholarsh 2003;35:61-6. http://www.ncbi.nlm.nih.gov/entrez/ query.fcgi? $\mathrm{cmd}=$ Retrieve $\& \mathrm{db}=$ PubMed\&list_uids $=12701528 \&$ $\mathrm{dopt}=\mathrm{Abstract}$ 